\title{
Potentials of telemedicine for green health care
}

\author{
Heinrich J. Audebert ${ }^{1 *}$, Thomas Meyer ${ }^{2}$ and Fabian Klostermann ${ }^{3}$ \\ 1 Center for Stroke Research, Charité Universitätsmedizin Berlin, Berlin, Germany \\ 2 Department of Neurology, Campus Virchow Klinikum, Charité Universitätsmedizin Berlin, Berlin, Germany \\ ${ }^{3}$ Department of Neurology, Campus Benjamin Franklin, Charité Universitätsmedizin Berlin, Berlin, Germany
}

Edited by:

Steven Levine, Mount Sinai School of Medicine, USA

\section{Reviewed by:}

Jeffrey A. Switzer, Medical College of

Georgia, USA

\section{*Correspondence:}

Heinrich J. Audebert, Center for Stroke Research, Charité Universitätsmedizin Berlin, Hindenburgdamm 30, 12200

Berlin, Germany.

e-mail: heinrich.audebert@charite.de
Neurological facilities are traditionally centered in academic hospitals and often far away from the patients' living area. Both, the transfer of patients to remote hospitals and inpatient treatment are associated with high energy consumption. Numbers of patients with neurological diseases are expected to increase along with the demographical changes and the environmental impact of neurological treatment should become a target for health policy, therefore. Positive effects have been demonstrated for the use of telemedicine by improving inpatient treatment in local community hospitals or avoiding hospital admissions via monitoring of complex diseases in outpatient settings. We discuss the potentials of telemedicine in the field of in-and outpatient neurological care as well as the need for more scientific evaluation on environmental impacts.

Keywords: telemedicine, teleneurology, telemonitoring, energy savings, environment protection
Health care is a key area of modern economies with growing numbers of employees and medical options. These positive developments are, however, associated with high energy costs whose prevention is hardly under debate up to date (unlike in many industrial sectors). This appears of particular note since the ecological burden of anthropogenic climate change certainly implies serious health risks, such as malnutrition or disease due to natural disasters or uncontrolled migration.

In this context, a consequent utilization of telecommunication technologies in healthcare (telemedicine) merits higher attention from medical providers and decision makers. This claim is also reasonable facing the demographical changes of western societies, implying an increasing number of neurovascular diseases and prevalence of degenerative, in particular, neurological dysfunctions over the next decades. Telemedicine may play a major role in reducing both environmental and economical costs in the future, at the same time as developing medical quality. This contribution illustrates the potentials of this approach with a special focus on applications in Neurology.

\section{STRENGTHENING OF LOCAL HOSPITAL TREATMENT}

Over the last decades, improved mobility has increased health related traffic, particularly in developed countries. In addition, under the growing pressure of both economic and high-quality medical treatment, many relatively small hospitals in rural areas have been closed to the advantage of more specialized centers in larger cities. As a consequence, this centralization goes along with high costs of patient transportation. Although the patient himself may only be transferred once, the commuting of next of kin between home and remote hospitals often accumulates thousands of miles.

Stroke care is an example for the area of conflict between centralization of medical institutions and the resulting remoteness of treatment. On the one hand, there is evidence that stroke unit care (Cochrane Collaboration, 2002) and specific treatments (Hacke et al., 2004; Vahedi et al., 2007) improve the prognosis of stroke patients. These treatment options require dedicated teams and specialized expertise, best available in academic hospitals. On the other hand, stroke is a common disease with highest incidence in elderly people and referral of all patients to few centralized facilities could easily swamp existing services and produce difficulties in near-home post stroke care.

Since stroke syndromes can be appropriately assessed via audiovisual examination, and brain imaging can easily be transferred via electronic transmission, Telestroke as a special form of telemedicine may help to overcome some of the shortcomings of regional hospitals by providing the expertise of established stroke centers on demand and within minutes (Levine and Gorman, 1999; Meyer et al., 2008). Using the Telestroke approach, intravenous thrombolysis in ischemic stroke has been shown to be safe and to be applied more frequently than without teleneurology consultation (Schwamm et al., 2004; Audebert et al., 2005a; Audebert and Schwamm, 2009b). Embedded in a comprehensive stroke network with dedicated stroke wards in community hospitals, the teletransfer of clinical information and brain images yields similar medical results as treatment in experienced stroke centers (Audebert et al., 2006a; Schwab et al., 2007). Particularly, telemedical collaboration does improve stroke prognosis in rural community hospitals without increasing the number of interhospital referrals (Audebert et al., 2009a,b). Specific stroke subtypes such as subarachnoid hemorrhages or malignant infarctions (Vahedi et al., 2007), which need complex diagnostic procedures or interventional treatments, may indeed require an interhospital transfer to specialized institutions, but this can be organized timely and selectively within telemedicine networks (Audebert et al., 2005b).

Other possible applications for Teleneurology in acute hospital settings include the management of epileptic seizures, the diagnostic work-up of unclear unconsciousness or remote evaluations of a patient's suitability for complex interventional treatments. 


\section{AVOIDING UNNECESSARY HOSPITAL ADMISSIONS}

Inpatient treatment in hospitals is associated with very high energy consumption. Indeed, the energy consumption per average hospital bed in Germany is above that of two modern detached houses (Fraunhofer Institute for Environmental, 2007). Thus, avoiding hospital admissions can help to reduce healthcare-related energy costs.

The practicability of this has been shown, e.g., using electronic transmission of vital parameters (telemonitoring) in patients with chronic heart failure (Cleland et al., 2005; Clark et al., 2007; Morguet et al., 2008), where alterations of body weight, heart rate and blood pressure were monitored as a basis of treatment modifications including medication changes. In this context, telemonitoring has been shown to avoid hospital admissions and to reduce mortality compared to usual care. Similar applications and effects have been reported for the management of diabetes mellitus (Barnett et al., 2006) or chronic respiratory failure (Vitacca et al., 2009).

Hospital admissions are associated with high carbon dioxide emissions when specialized facilities are very distant of the place of patients' home. This is particularly true in rural areas or on remote islands.

In the field of Neurology, telemonitoring could improve the management of several chronic diseases and might substitute part of the hospital admissions related to the respective conditions. For example, video-based telemonitoring has already been implemented in the therapy of Parkinson's disease (PD). In advanced $\mathrm{PD}$, daily fluctuations of mobility often become a major problem, but the rapidly changing motor states of patients are mostly difficult to assess during short outpatient visits. On the other hand, this particular condition often requires complex adjustments of medication, so that it is a frequently mentioned reason of hospital admissions in PD patients. Against this background, real-time or asynchronous tele-assessment can convey a representative picture of the patient's condition in his natural surroundings. It further allows for many therapeutic changes which can be realized in constant touch with the patient who-being relatively immobile - does not have to leave his home environment. First experiences with this approach are promising (Hubble et al., 1993; Biglan et al., 2009) and further evaluation is currently underway.

Other examples comprise the observation of patients in there home environment (telesurveillance) for patients with amyotrophic lateral sclerosis (ALS) and other motor neuron diseases. ALS is regarded as a paradigmatic disorder for patient autonomy and complex home care management including out-of-hospital mechanical ventilation, enteral feeding and implementation of advanced communication systems. The combination of telesurveilance, telemonitoring and interactive telecommunication via telephone, computer or videoconference is a powerful approach that may support patients in handling their condition and reduce their fear of respiratory failure (Vitacca et al., 2010). A pilot trial testing the feasibility of a telesurveillance program combined with educational support is currently underway (NCT00613899). This study is aiming to test whether telesurveillance may increase the

\section{REFERENCES}

Audebert, H. J., Kukla, C., Clarmann, v. C., Kuhn, J., Vatankhah, B., Schenkel, J., Ickenstein, G. W., Haberl, R. L., and Horn, M. (2005a). Telemedicine for safe and extended use of thrombolysis in stroke: the Telemedic Pilot Project for Integrative Stroke Care (TEMPiS) in Bavaria. Stroke 36, 287-291.

Audebert, H. J., Wimmer, M. L., Hahn, R., Schenkel, J., Bogdahn, U., Horn, M., and Haberl, R. L. (2005b). Can tele-

safety of home ventilation while in hospital care is reduced. The immobility of patients, the physical and economic burden of patient transfer and lack of regional ALS centers make teleconsulting during patient follow-up attractive. Web based solutions for patient self assessment and management have been developed (www.patientslikeme.com; www.alshome.de).

Another application of telemedicine for neurologists consists of attending specialist outpatient clinics without traveling to remote clinic locations. Such a Teleneurology service has been demonstrated to substantially reduce traveling (Smith et al., 2007). Similar utilizations of telemedicine have been shown in members of multidisciplinary cancer teams (Lewis et al., 2009) or in patients with head and neck cancer who live in the Shetland Islands (Dorrian et al., 2009).

\section{PROFESSIONAL ACTIVITIES}

Neurology is an evolving science, demanding continuous updating and interchange of professional knowledge. Better connections, cheaper flights and a broad spectrum of conferences seem to meet this goal, but - of course -nobody will deny the ecological burden of such developments. With modern telecommunication, e.g., using videoconference links as already done in other areas of international business, some part of conference traveling could certainly be avoided. It may first of all be a matter of getting used to these still unfamiliar techniques to even enhance medical information exchange. Naturally, physical conference participation will remain necessary on specific occasions but, principally, the reputation of health professionals should rather be based on knowledge and skills than on miles in frequent flyer programs.

\section{RESEARCH}

It is difficult to estimate the impact of medical progress on environmental damage and to weigh these effects against medical outcomes. However, as with economic consequences, our long-term responsibility calls for also considering environmental perspectives of medical development. In this sense, the assessment of net effects of telemedicine strategies on the environment may become a future field of investigation, just as a more wide-spread use of this technology in clinical research trials appears desirable.

\section{SUMMARY}

As a professional group, physicians have not traditionally considered the environmental impact of healthcare, nor has this been in the focus of medical research so far. Of course, high energy strategies will always be necessary, if they can improve patient outcomes, but we should minimize negative environmental impacts whenever possible. In this regard, a broader implementation of telemedicine appears a promising and long-ranging option.

\section{ACKNOWLEDGMENTS}

Parts of the manuscript were originally drafted in a collaboration of Heinrich J. Audebert and Peter Langhorne.

medicine contribute to fulfill WHO Helsingborg Declaration of specialized stroke care? Cerebrovasc. Dis. 20, 362-369.

Audebert, H. J., Kukla, C., Vatankhah, B., Gotzler, B., Schenkel, J., Hofer, S.,
Furst, A., and Haberl, R. L. (2006a). Comparison of tissue plasminogen activator administration management between Telestroke Network hospitals and academic stroke centers: the Telemedical Pilot Project for 
Integrative Stroke Care in Bavaria/ Germany. Stroke 37, 1822-1827.

Audebert, H. J., Schenkel, J., Heuschmann, P. U., Bogdahn, U., and Haberl, R. L. (2006b). Effects of the implementation of a telemedical stroke network: the Telemedic Pilot Project for Integrative Stroke Care (TEMPiS) in Bavaria, Germany. Lancet Neurol. 5, 742-748.

Audebert, H. J., Schultes, K., Tietz, V., Heuschmann, P. U., Bogdahn, U., Haberl, R. L., and Schenkel, J. (2009a). Long-term effects of specialized stroke care with telemedicine support in community hospitals on behalf of the Telemedical Project for Integrative Stroke Care (TEMPiS). Stroke 40, 902-908.

Audebert, H. J., and Schwamm, L. (2009b). Telestroke: scientific results. Cerebrovasc. Dis. 27(Suppl 4), 15-20.

Barnett, T. E., Chumbler, N. R., Vogel, W. B., Beyth, R. J., Qin, H., and Kobb, R. (2006). The effectiveness of a care coordination home telehealth program for veterans with diabetes mellitus: a 2-year follow-up. Am. J. Manag. Care 12, 467-474.

Biglan, K. M., Voss, T. S., Deuel, L. M., Miller, D., Eason, S., Fagnano, M., George, B. P., Appler, A., Polanowicz, J., Viti, L., Smith, S., Joseph, A., and Dorsey, E. R. (2009). Telemedicine for the care of nursing home residents with Parkinson's disease. Mov. Disord. 24, 1073-1076.

Clark, R. A., Inglis, S. C., McAlister, F. A., Cleland, J. G., and Stewart, S. (2007). Telemonitoring or structured telephone support programmes for patients with chronic heart failure: systematic review and meta-analysis. BMJ 334, 942.
Cleland, J. G., Louis, A. A., Rigby, A. S., Janssens, U., and Balk, A. H. (2005). Noninvasive home telemonitoring for patients with heart failure at high risk of recurrent admission and death: the Trans-European Network-Home-Care ManagementSystem(TEN-HMS) study. J. Am. Coll. Cardiol. 45, 1654-1664.

Cochrane, Collaboration. (2002). Organised inpatient (stroke unit) care for stroke. Cochrane Database Syst. Rev. CD000197.

Dorrian, C., Ferguson, J., Ah-See, K., Barr, C., Lalla, K., van der, P. M., McKenzie, L., and Wootton, R. (2009). Head and neck cancer assessment by flexible endoscopy and telemedicine. $J$. Telemed. Telecare 15, 118-121.

Fraunhofer Institute for Environmental, Safety and Energy Technology UMSICHT. (2007). Energiesparen im Krankenhaus. Available at http://www. rhombos.de/shop/a/show/story/?796 Last access. 16-11-2007.

Hacke, W., Donnan, G., Fieschi, C., Kaste, M., von Kummer, R., Broderick, J. P., Brott, T., Frankel, M., Grotta, J. C., Haley, E. C.Jr., Kwiatkowski, T., Levine, S. R., Lewandowski, C., Lu, M., Lyden, P., Marler, J. R., Patel, S., Tilley, B. C., Albers, G., Bluhmki, E., Wilhelm, M., and Hamilton, S. (2004). Association of outcome with early stroke treatment: pooled analysis of ATLANTIS, ECASS, and NINDS rt-PA stroke trials. Lancet 363, 768-774.

Hubble, J. P., Pahwa, R., Michalek, D. K., Thomas, C., and Koller, W. C. (1993). Interactive video conferencing: a means of providing interim care to Parkinson's disease patients. Mov. Disord. 8, 380-382.

Levine, S. R., and Gorman, M. (1999). "Telestroke": the application of telemedicine for stroke. Stroke 30 464-469.

Lewis, D., Tranter, G., and Axford, A. T. (2009). Use of videoconferencing in Wales to reduce carbon dioxide emissions, travel costs and time. $J$. Telemed. Telecare 15, 137-138.

Meyer, B. C., Raman, R., Hemmen, T., Obler, R., Zivin, J.A., Rao, R., Thomas, R. G., and Lyden, P.D. (2008). Efficacy of site-independent telemedicine in the STRokE DOC trial: a randomised, blinded, prospective study. Lancet Neurol. 7, 787-795.

Morguet, A. J., Kuhnelt, P., Kallel, A., Jaster, M., and Schultheiss, H. P. (2008) Impact of telemedical care and monitoring on morbidity in mild to moderate chronic heart failure. Cardiology 111, 134-139.

Schwab, S., Vatankhah, B., Kukla, C. Hauchwitz, M., Bogdahn, U., Furst, A., Audebert, H. J., and Horn, M. (2007). Long-term outcome after thrombolysis in telemedical stroke care. Neurology 69, 898-903.

Schwamm, L. H., Rosenthal, E. S., Hirshberg, A., Schaefer, P. W., Little, E. A., Kvedar, J. C., Petkovska, I., Koroshetz, W. J., and Levine, S. R. (2004) Virtual TeleStroke support for the emergency department evaluation of acute stroke. Acad. Emerg. Med. 11, 1193-1197.

Smith, A. C., Patterson, V., and Scott, R. E. (2007). Reducing your carbon footprint: How telemedicine helps. $B M$ J $335,1060$.

Vahedi, K., Hofmeijer, J., Juettler, E. Vicaut, E., George, B., Algra, A., Amelink, G. J., Schmiedeck, P., Schwab, S., Rothwell, P. M., Bousser, M. G., van der Worp, H. B., and Hacke, W. (2007). Early decompressive surgery in malignant infarction of the middle cerebral artery: a pooled analysis of three randomised controlled trials. Lancet Neurol. 6, 215-222.

Vitacca, M., Bianchi, L., Guerra, A., Fracchia, C., Spanevello, A., Balbi, B. and Scalvini, S. (2009). Tele-assistance in chronic respiratory failure patients: a randomised clinical trial. Eur. Respir. J. 33, 411-418.

Vitacca, M., Comini, L., Tentorio, M., Assoni, G., Trainini, D., Fiorenza, D., Morini, R., Bruletti, G., and Scalvini, S. (2010). A pilot trial of telemedicine-assisted, integrated care for patients with advanced amyotrophic lateral sclerosis and their caregivers. $J$. Telemed. Telecare 16, 83-88.

Conflict of Interest Statement: The authors declare that the research was conducted in the absence of any commercial or financial relationships that could be construed as a potential conflict of interest.

Received: 19 April 2010; paper pending published: 11 May 2010; accepted: 21 May 2010; published online: 02 August 2010.

Citation: Audebert HJ, Meyer T and Klostermann F (2010) Potentials of telemedicine for green health care. Front. Neur. 1:10. doi: 10.3389/fneur.2010.00010

This article was submitted to Frontier in Teleneurology, a specialty of Frontiers in Neurology.

Copyright $\odot 2010$ Audebert, Meyer and Klostermann. This is an open-access article subject to an exclusive license agreement between the authors and the Frontiers Research Foundation, which permits unrestricted use, distribution, and reproduction in any medium, provided the original authors and source are credited. 